\title{
La ginecomastia tuberosa, una entidad poco reconocida en la práctica quirúrgica actual
}

\author{
Tuberous gynecomastia, an entity that is not very well \\ recognized in current surgical practice
}

\author{
José E Telich-Tarriba, ${ }^{*, \ddagger}$ Daniel Garza-Arriaga, ${ }^{\S}$ Ahilyn Leyva-Vázquez, ${ }^{*, \neq}$ José Telich-Vidal*,‡ \\ Citar como: Telich-Tarriba JE, Garza-Arriaga D, Leyva-Vázquez A, Telich-Vidal J. La ginecomastia tuberosa, una entidad poco \\ reconocida en la práctica quirúrgica actual. Acta Med. 2020; 18 (4): 414-417. https://dx.doi.org/10.35366/97270
}

\section{Resumen}

La ginecomastia es una proliferación benigna del tejido glandular mamario en hombres, resultando en aumento de volumen en la región pectoral. Tiene una amplia variedad de causas, las cuales se clasifican como fisiológicas o patológicas; en el fondo la fisiopatología es multifactorial, dependiendo de una mayor producción de estrógenos periféricos, o menor producción o sensibilidad a andrógenos. Existen distintas clasificaciones de ginecomastia en la literatura internacional, las más empleadas son las de Simon y Rohrich, las cuales toman en cuenta principalmente el volumen mamario y la presencia o ausencia de ptosis glandular. En varones, la ginecomastia tuberosa se caracteriza por la presencia de exceso cutáneo en el polo inferior, un anillo de constricción fibroso periareolar y crecimiento glandular vertical. Es un diagnóstico poco común en la práctica clínica, por lo que su epidemiología es desconocida. El objetivo del tratamiento quirúrgico es lograr una apariencia normal del tórax masculino con la cicatriz más pequeña posible. El tratamiento quirúrgico de la ginecomastia requiere un abordaje individualizado, en ocasiones combinando liposucción y mastectomía, mientras que en la mama tuberosa masculina requiere que se aborde la relativa redundancia de la piel, incluso cuando el exceso de tejido es menor.

Palabras clave: Ginecomastia, mama tuberosa, masculino, liposucción.

\section{Abstract}

Gynecomastia is a benign proliferation of mammary glandular tissue in men, resulting in increased volume in the pectoral region. It has a wide variety of causes, which are classified as physiological or pathological; basically, the pathophysiology is multifactorial, depending on a higher production of peripheral estrogens, or less production and sensitivity to androgens. There are different classifications of gynecomastia in the international literature, the most widely used are those of Simon and Rohrich, which mainly take into account breast volume and the presence or absence of glandular ptosis. In men, tuberous gynecomastia is characterized by the presence of cutaneous excess in the lower pole, a periareolar fibrous constriction ring and vertical glandular growth. It is a rare diagnosis in clinical practice, so its epidemiology is unknown. The goal of surgical treatment is to achieve a normal appearance of the male chest with the smallest possible scar. The surgical treatment of gynecomastia requires an individualized approach, sometimes combining liposuction plus mastectomy, while in the male tuberous breast it requires that the relative redundancy of the skin be addressed even when there is relatively less tissue excess.

Keywords: Gynecomastia, tuberous breast, male, liposuction.
* Departamento de Cirugía Plástica y Reconstructiva, Hospital Ángeles Pedregal.

‡ Postgrado en Cirugía Estética, Facultad Mexicana de Medicina Universidad La Salle.

§ Departamento de Cirugía Plástica y Reconstructiva, Fundación del Cáncer de Mama A.C.

Ciudad de México, México.
Correspondencia:

Dr. José Telich Tarriba

Correo electrónico: jtelich@prodigy.net.mx

Aceptado: 01-05-2020.

www.medigraphic.com/actamedica

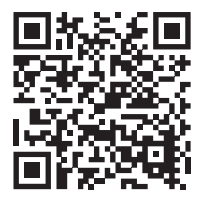




\section{INTRODUCCIÓN}

La ginecomastia se define como una proliferación benigna del tejido glandular mamario en hombres, la cual provoca aumento de volumen en la región pectoral. Tiene una amplia variedad de causas y se clasifican como fisiológicas o patológicas; en el fondo, la fisiopatología es multifactorial, dependiendo de una mayor producción de estrógenos periféricos, o menor producción o sensibilidad a andrógenos. ${ }^{1}$

Se trata de una entidad relativamente común con una prevalencia estimada que varía del 4 al $64 \%$ en el ámbito internacional $y$, de acuerdo con los resultados de la encuesta ISAPS 2018, su corrección es el procedimiento estético más realizado en hombres tanto en México como en el resto del mundo. ${ }^{2}$

La ginecomastia tuberosa es una entidad que suele mencionarse poco en la literatura y no se incluye en las clasificaciones; la mama tuberosa se caracteriza por la presencia de un anillo de constricción y expansión del complejo areola-pezón; aparece aproximadamente en cinco de cada 10,000 mujeres. ${ }^{3,4}$

En hombres, la ginecomastia tuberosa se caracteriza por la presencia de exceso cutáneo en el polo inferior, un anillo de constricción fibroso periareolar y crecimiento glandular vertical. Es un diagnóstico poco común en la práctica clínica, por lo que su epidemiología es desconocida; Cannistra reportó siete casos en una serie de 58 pacientes con ginecomastia, obtuvo como resultado una prevalencia del $12.06 \%$, suele agruparse dentro del subtipo IIIb de la clasificación de Simon. ${ }^{1}$

El objetivo de esta investigación es presentar un caso de ginecomastia tuberosa, su manejo quirúrgico y realizar una revisión de la literatura.

\section{CASO CLÍNICO}

Hombre de 15 años, sin antecedentes médicos o quirúrgicos de relevancia, quien se presentó con un historial de crecimiento mamario bilateral de dos años de evolución, el cual fue manejado inicialmente en otra institución con tamoxifeno (10 mg cada 12 horas) durante tres meses, sin notar mejoría, acude a consulta para segunda opinión; a la exploración clínica dirigida se encuentra crecimiento mamario bilateral, simétrico con protrusión areolar. Los estudios paraclínicos que consisten en perfil hormonal, niveles de estradiol, testosterona, progesterona, $\mathrm{LH}$, hormona de crecimiento, perfil tiroideo, prolactina, DHEA, hCG todos sin evidencia de alteraciones hormonales; asimismo, en el interrogatorio dirigido tampoco se evidenció tratamiento con antiulcerosos como cimetidina, ranitidina u otro medicamento del grupo, el ultrasonido mamario reportó presencia de tejido glandular mamario hipertrófico retroareolar, sin alteraciones estructurales (Figura 1).

Se decide manejo quirúrgico, con resección de tejido mamario bilateral. Se somete a tratamiento mixto (liposucción y mastectomía periareolar). En principio, se infiltraron en cada lado $100 \mathrm{~mL}$ de solución de Klein por medio de puertos axilares e inferolaterales. Se realizó liposucción con jeringas de $60 \mathrm{~mL}$ con cánulas de punta roma de $2 \mathrm{~mm}$, utilizando los mismos puertos hasta aislar el tejido glandular del tejido graso circundante.

Después se realizó un abordaje periareolar inferior que resecó en su totalidad el tejido glandular, y permitió identificar la presencia de un anillo fibroso periareolar y fibrosis en el polo inferior de la glándula, compatible con la definición del diagnóstico de mama tuberosa. Al igual que con las mamas tuberosas femeninas, la base constreñida se liberó con electrocauterio realizando incisiones radiales del tejido fibroso residual del seno debajo de la areola, con resección de tejido mamario aproximadamente de $118 \mathrm{~g}$, el cual se envía a patología con reporte histopatológico de tejido mamario con ginecomastia.

Después de verificar hemostasia, se colocaron drenajes de succión y se realizó el cierre por planos con sutura intradérmica. Los drenajes fueron retirados al tercer día postoperatorio y el sujeto empleó una prenda de compresión durante cuatro semanas. Se realizó seguimiento postquirúrgico con retiro de puntos a las dos semanas, evolución después de uno, tres y seis meses con adecuada retracción del tejido y cicatrización. Obtuvimos el reporte de histopatología que reporta tejido mamario bilateral con ginecomastia.

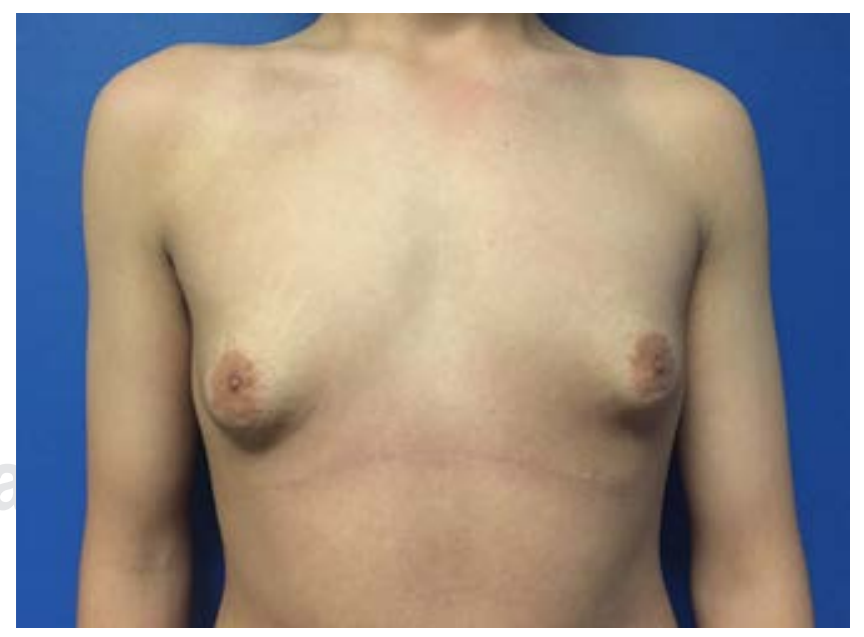

Figura 1: Imagen clínica preoperatoria del paciente. Se evidencia el aumento de volumen del complejo areola-pezón debido a la herniación glandular, redundancia cutánea en la región pectoral y constricción del polo glandular inferior. 


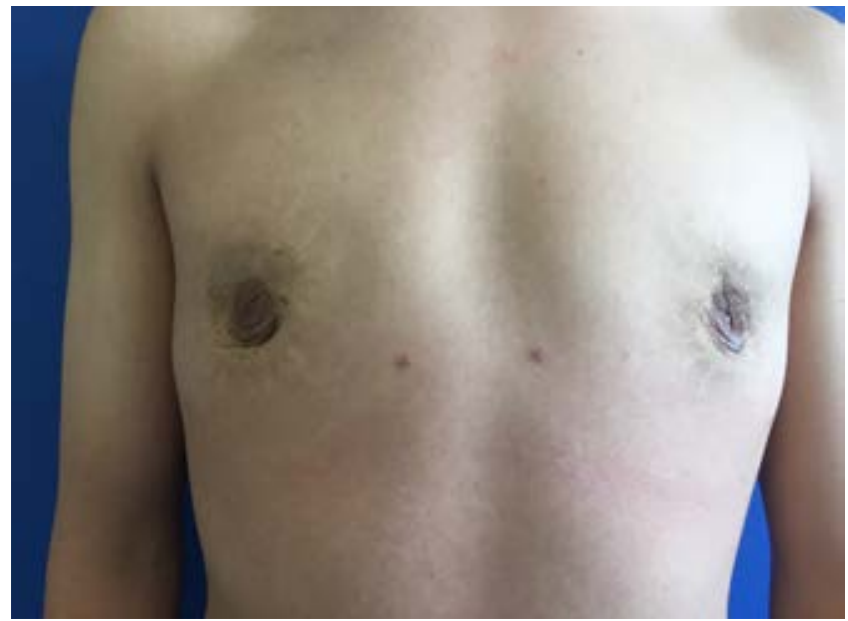

Figura 2: Fotografía postoperatoria en la que se observa corrección del volumen mamario, con adecuada retracción cutánea y viabilidad de complejos areola-pezón.

En la actualidad, el sujeto se encuentra con adecuada evolución sin evidencia de crecimiento de tejido mamario glandular ni graso, así como una adecuada cicatrización y sin evidencia de anillo de constricción de mama tuberosa, presentando resultado estético satisfactorio (Figura 2).

\section{DISCUSIÓN}

La ginecomastia es una alteración morfoestructural benigna de la región mamaria que afecta a los hombres, la palabra deriva de las raíces griegas gyné (mujer) y mastós (glándula mamaria). Es causada por una hipertrofia parenquimatosa con distorsión cutánea de la cubierta de la piel de una o ambas mamas. Se considera una afección común que causa importantes alteraciones emocionales y sociales. ${ }^{1}$

La incidencia difiere en muchos informes y varía entre el 4 y $69 \%$ en casos con tejido mamario palpable, puede presentarse desde los 10 años, con un pico de incidencia entre las edades de 13 y 14 años, esto es seguido por una disminución de la incidencia en la adolescencia tardía, sólo el 10\% de los casos de ginecomastia persistente tienen 17 años. $^{5}$

En la mayoría de los casos, se desconoce la etiología, ésta puede asociarse con anomalías congénitas como en el síndrome de Klinefelter, a desequilibrios hormonales como insuficiencia testicular o anorquia, enfermedades hepáticas, tiroideas y renales, también tratamientos farmacológicos, como lo son los tratamientos para el cáncer prostático y los inhibidores $\mathrm{H} 2$. Se acepta como posible causa un desbalance entre la actividad de los estrógenos y los andrógenos a nivel glandular, a pesar de que en la mayoría de los estudios publicados no se encuentran alte- raciones en los niveles de hormonas tanto de andrógenos como de estrógenos, comparándolos con los niños púberes sin ginecomastia; de ahí que se sugiera que en estos niños existiría una mayor sensibilidad de las células mamarias a los niveles normales de estrógenos circulantes. Otra teoría sugiere la existencia de un predominio estrogénico al inicio puberal cuando comienza la secreción nocturna pulsátil de LH por la hipófisis. La obesidad puede incrementar el riesgo de desarrollo de ginecomastia. ${ }^{1}$

Simon y colaboradores clasificaron clínicamente en 1973 la ginecomastia de la siguiente manera: (I) poco aumento del volumen mamario sin exceso cutáneo; (II) aumento moderado del volumen mamario sin (Ila) o con (Ilb) exceso cutáneo; (III) aumento significativo del volumen mamario con un exceso cutáneo similar a la ptosis mamaria (en especial en personas obesas). La mama tuberosa masculina es un subtipo en el esquema de clasificación de Simon y su equipo caracterizado como IIIb. ${ }^{3,6}$

En 1976, Rees y Aston utilizaron por primera vez el término de "mama tuberosa", que se caracteriza por un anillo de restricción en la base de la mama, que está ausente o es deficiente en la dimensión vertical y horizontal. De etiología idiopática, el complejo areola-pezón está muy desarrollado, con una hernia del tejido mamario en la areola. Pacifico y colaboradores reportaron que la causa es un adelgazamiento en la piel areolar con respecto a la piel circundante..$^{4,7,8}$ Es una malformación del parénquima que se ha descrito ampliamente, esta alteración sólo aparece durante el crecimiento, a menudo involucra ambos senos y tiene una alta incidencia de asimetría. Otra hipótesis es que se debe a un engrosamiento anormal de la fascia corporis que conduce a una alteración en el desarrollo glandular, esta estenosis limita el crecimiento del parénquima horizontal, llevando finalmente a la mama a adquirir el aspecto clínico típico de mama tuberosa. ${ }^{9}$

En grados avanzados, puede ser una fuente de estigma y preocupación, lo que lleva a la necesidad de corrección quirúrgica para mejorar la apariencia estética. Por lo general, afecta a las mujeres durante la pubertad y hay pocos casos reportados en los hombres y estos pueden estar asociados con ginecomastia con las mismas características que la condición correspondiente en las mujeres. ${ }^{5}$

De acuerdo con la clasificación descrita por Von Heimburg, la mama tuberosa se clasifica en: tipo I hipoplasia del cuadrante medial inferior; tipo II hipoplasia de los cuadrantes medial y lateral inferior, suficiente piel en la región aural; tipo III hipoplasia de los cuadrantes mediales y laterales inferiores, deficiencia de la piel en la región sub-areolar; y tipo IV constricción mamaria severa, base mamaria mínima. ${ }^{10}$ El cuadro clínico no encaja fácilmente en la clasificación sugerida por Simon y su equipo, en esta condición es necesaria la reducción 
de la piel, incluso si existe un grado relativamente bajo de hipertrofia mamaria. Generalmente se observan ptosis y herniación significativa del tejido mamario en el complejo areola-pezón.

El tratamiento quirúrgico de la ginecomastia se debe considerar en adolescentes varones que presentan un aumento persistente de las mamas después de un periodo de observación de al menos 12 meses, mastalgia, hipersensibilidad $y / 0$ malestar psicosocial significativo. ${ }^{5}$

El objetivo del tratamiento quirúrgico es lograr una apariencia normal del tórax masculino con la cicatriz más pequeña posible. El tratamiento quirúrgico de la ginecomastia requiere un abordaje individualizado, la técnica más utilizada es la mastectomía subcutánea que implica la resección directa del tejido glandular, mediante un abordaje periareolar o transareolar, con o sin liposucción asociada. La escisión de la piel es necesaria para casos más avanzados. ${ }^{3,11}$

La mama tuberosa masculina requiere un enfoque individualizado en el que se aborde la ginecomastia, así como la relativa redundancia de la piel incluso cuando el exceso de tejido es menor. Los procedimientos quirúrgicos realizados van desde la mastectomía simple hasta la resección del anillo de constricción a través de un rayado periférico del mismo, combinado con liposucción o resección glandular, y en casos más avanzados en los que se presente hipertrofia severa con importante ptosis, resección de piel a través de una mastopexia y reposicionamiento de complejo areola-pezón. ${ }^{12}$

La decisión de realizar la cirugía depende del grado en que esta condición haya afectado la calidad de vida y del deseo de cada persona de corrección estética. En nuestro, centro tratamos la ginecomastia simple con una incisión periareolar y glandulectomía subcutánea combinada con lipoaspiración.

En el caso que presentamos, optamos por añadir a la técnica habitual la disrupción del anillo fibroso periareolar que estaba presente, por lo que el cirujano debe conocer las diferentes opciones técnicas para personalizar la reparación según el tipo de deformidad. Nosotros recomendamos la liposucción en todos los casos, como un tratamiento para la ginecomastia leve, un complemento de la escisión quirúrgica o una maniobra primaria de reducción de volumen antes de la corrección quirúrgica.

\section{CONCLUSIÓN}

A pesar de que la ginecomastia es un padecimiento frecuentemente observado en varones, la mama tuberosa masculina es una condición infrecuente en nuestro medio, a menudo se diagnostica de manera errónea en mujeres y hombres, por lo tanto, su prevalencia es difícil de determinar. Es importante saber que requiere estrategias de tratamiento específicas para cada uno de los casos, como la disrupción del anillo fibroso y el manejo del complejo areola-pezón para lograr resultados satisfactorios a largo plazo, así como un adecuado manejo del tejido mamario excedente y en ocasiones de la piel. En este caso, optamos por un manejo combinado buscando siempre obtener el mejor resultado funcional y estético. Se muestran las imágenes preparatorias en donde se evidencian las alteraciones morfológicas, no se muestran fotografías postquirúrgicas debido a que nuestro paciente vive en el extranjero; sin embargo, nos hemos comunicado con él y nos informa que se encuentra en óptimas condiciones.

\section{REFERENCIAS}

1. Cannistra C, Piedimonte A, Albonico F. Surgical treatment of gynecomastia with severe ptosis: periareolar incision and dermal double areolar pedicle technique. Aesth Plast Surg. 2009; 33: 834-837.

2. ISAPS Global Survey Results 2018. (Accesado el 05/04/2020) Available in: https://www.isaps.org/wp-content/uploads/2019/12/ ISAPS-Global-Survey-Results-2018-new.pdf.

3. Klinger M, Caviggioli F, Klinger F, Villani F, Arra E, Di Tommaso L. Tuberous breast: Morphological study and overview of a borderline entity. Can J Plast Surg. 2011; 19 (2): 42-44.

4. Martin G, García-Vilanova A, Sanz E, Fuster C, Vicente Roig J. Mama tuberosa en el varón. Cirugía Española. 2012; 90 (9): 606-607.

5. Hamilton S, Gault D. The tuberous male breast. Br J Plast Surg. 2003; 56 (3): 295-230.

6. Simon BE, Hoffman S, Kahn S. Classification and surgical correction of gynecomastia. Plast Reconstr Surg. 1973; 51 (1): 48-52.

7. Pacifico MD, Kang NV. The tuberous breast revisited. J Plast Reconstr Aesthet Surg. 2007; 60:455-464.

8. Rees TD, Aston SJ. The tuberous breast. Clin Plast Surg. 1976;3(2):339-347.

9. Greydanus D, Matytsina L, Gains M. Breast disorders in children and adolescents. Prim Care Clin Office Pract. 2006; 33: 455-502.

10. Heimburg D, Exner K, KruftS, Lemperle G. The tuberous breast deformity: classification and treatment. Br J Plast Surg. 1996; 49 (6): 339-345.

11. Nahabedian M. Breast deformities and mastopexy. Plast Reconstr Surg. 2011; 127: 91e-102e.

12. Monteiro D, Horta R, Amarante J, Silva Á, Silva P. Tuberous male breast: assessment and esthetic correction. Breast J. 2015; 21 (6): 696-698. 